\title{
Quasi-Non-Destructive Evaluation of Yield Strength Using Neural Networks
}

\author{
G. Partheepan, D. K. Sehgal, and R. K. Pandey \\ Department of Applied Mechanics, Indian Institute of Technology Delhi, Hauz Khas, New Delhi 110016, India \\ Correspondence should be addressed to D. K. Sehgal, profsehgal@gmail.com
}

Received 26 January 2011; Accepted 17 April 2011

Academic Editor: Ping Feng Pai

Copyright () 2011 G. Partheepan et al. This is an open access article distributed under the Creative Commons Attribution License, which permits unrestricted use, distribution, and reproduction in any medium, provided the original work is properly cited.

The objective of this paper is to delineate a method for determining the yield strength of a material in a virtually nondestructive manner. Conventional test methods for predicting the yield strength require the removal of large material samples from the inservice component, which is impractical. In this paper, the power of neural networks in predicting the yield strength from the data obtained by conducting tension test on newly developed dumb-bell-shaped miniature specimen is demonstrated using the self-organizing capabilities of the ANN. The input to the neural network is the breakaway load obtained from the miniature test, and the output obtained from the model is yield strength value. The value of the yield strength estimated by neural network is found to be in good agreement ( $<5 \%$ error) with that of the actual value from the standard test. The neural network models are convenient and powerful tools for practical applications in solving various problems in engineering.

\section{Introduction}

In the past, designers/engineers avoided material failure by designing the structures for stresses well below the yield strength of the material. However, when the same approach was used with high strength materials under extreme conditions, there were many catastrophic failures [1]. The material behavior of in-service structural components is changing due to in service loading, aging, irradiation, and other adverse conditions leading to embrittlement, which requires an in situ monitoring of the materials' state. It is not feasible to assess the degraded properties of these equipments and components by performing standard destructive mechanical tests, because this would mean damaging of equipments and rendering them nonfunctional in addition to long shut down [2]. In order to determine material parameters at various locations, for example, in weldments or gradient materials, the size of the material taken out for a test specimen should be very small but representative. Over the years, the subsize specimen test and miniature specimen test techniques have been evolved to estimate the various mechanical properties without seriously affecting the functionality of the components. In this respect, the small punch test, ball indentation test, disk bend test, shear punch test, and many other tests are reported for evaluation of degraded material properties of in-service components [3].

A variety of approaches have been employed to determine mechanical properties from small disks and coupons. The earliest use of the $3 \mathrm{~mm}$ diameter disk specimen was an attempt by Huang et al. [4] to assess the tensile ductility of a set of irradiated steels. They used simply supported TEM disks of $3 \mathrm{~mm}$ diameter, which were displaced by a spherical tipped indenter. Mao and Takahashi [5] and Mao et al. [6] investigated the deformation behaviour using small punch test. Kullen et al. [7] employed a shear punch test technique to determine the mechanical properties of neutron irradiated $9 \mathrm{Cr}-1 \mathrm{Mo}$ and $12 \mathrm{Cr}-1 \mathrm{Mo}$ steels. Pandey and Bhowmick [8] further used the disk bend test specimen to predict tensile properties and fracture toughness ( $J_{\text {IC }}$ ) for a number of steels. Recently, Husain [9] employed small punch test on different materials having varieties of strength to establish a general relationship between the data obtained from small punch test and the yield strength.

The developments in artificial intelligence made the researchers to have a look into the solution of nonlinear problems in physical and mechanical properties of metal alloys [10]. The field of ANN was born in an attempt to 
solve the complex problems that are difficult for conventional computers or otherwise. Their capacity to identify the underlying functional relationship in the data, while neglecting noisy and less significant input data strongly supports the applicability of such methods for prediction of material properties and other problems in engineering.

Recently, in material science and engineering fields, the researchers have used neural network models to predict the mechanical properties of materials. Ozerdem and Kolukisa [11] used $\mathrm{Cu}-\mathrm{Sn}-\mathrm{Pb}-\mathrm{Zn}-\mathrm{Ni}$ (wt\%) contents as input to the neural network to get the yield strength value of cast alloys. Similar exercise was performed using $\mathrm{C} \%, \mathrm{Si} \%$, and $\mathrm{Mn} \%$ contents as inputs to predict the yield strength of carbon steel bars [12].

Forouzan and Akbarzadeh [13] predicted the effect of thermomechanical parameters such as preheating time and temperature, finish rolling temperature and the final annealing temperature on mechanical properties such as yield strength, ultimate tensile stress, and elongation using neural network. ANN was used to predict the mechanical properties of forged TC11 titanium alloy by using the deformation temperature and the true strain as the inputs to the network [14]. In the same manner, Guo and Sha [15] modelled the correlation between processing parameters and properties of maraging steels using artificial neural network. The input parameters of their neural network model consist of alloy composition, processing parameters vis-à-vis cold deformation degree, ageing temperature and ageing time, and working temperature. The outputs obtained from ANN model include ultimate tensile strength, yield strength, elongation, and reduction in area. Also, similar exercise were performed for titanium alloys [16]. The effect of alloy composition, microstructure and work temperature on tensile properties of gamma-based titanium aluminides also studied using artificial neural networks [17]. On the other hand, Huang et al. [18] used ANN to predict the flexural strength and fracture toughness of ceramic tool materials. Zhang et al. [19] employed ANN model for the prediction of properties of composite materials. Zeng et al. [20] developed an expert system using neural network to identify cheaper sintering process to efficiently achieve the desired mechanical properties with respect to cost and time. ANN was also used to predict ductile properties of cast iron [21] and mechanical properties of annealed thin strip [22]. Huber and Tsakmakis [23] performed spherical indentation test to obtain constitutive properties from the resultant test data using neural network. They obtained a data base for the training and validation of the neural network by carrying out numerous finite element simulations using commercially available ABAQUS code, for various sets of material parameters. Abendroth and Kuna [24] used feedforward neural network model to find the material properties and tested this on three steels using small punch test.

From [25], it was noted that different test techniques have their own advantages and limitations. in the present investigation, an ANN model is developed using the miniature test load-elongation diagram to obtain the yield strength of materials. In the present research a dumb-bell-shaped miniature specimen has been designed and used to conduct

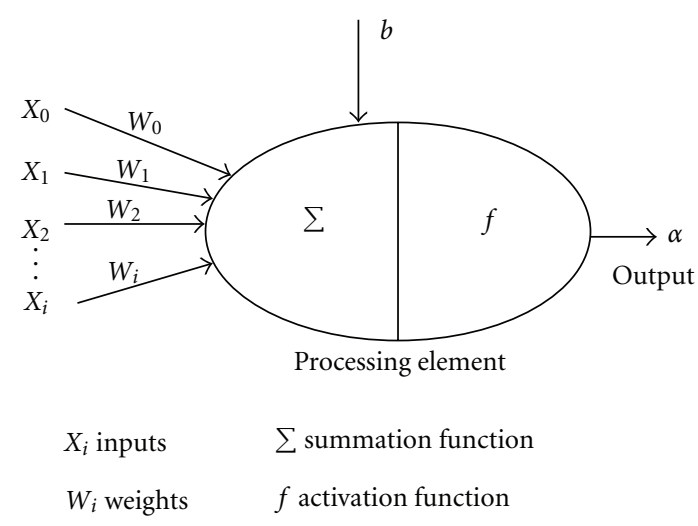

FIGURE 1: Schematic of an artificial neuron.

the miniature test through the experimental setup described elsewhere [26]. the miniature specimen is fabricated from the material taken out from the structure for which the properties are to be determined. Then, the miniature specimen test is conducted and the load-elongation diagram along with the yield load is obtained. Then, using the trained ANN model and the yield load, the yield strength of the structure can be obtained.

\section{Neural Network Model}

Neural Network models belong to the class of data driven approaches instead of model-driven approaches [27]. Their ability to learn by example makes artificial neural networks very flexible and powerful. Therefore, neural networks have been intensively used for solving regression and classification problems in many fields. Recently, neural networks have been used in the areas that require computational techniques such as pattern recognition, optical character recognition, predicting outcomes, and problem classification, prediction models for mechanical properties of materials [28].

\section{Background of Ann}

The basic units of neural network are the artificial neuron as shown in Figure 1. Neural networks analyze data by passing it through several simulated processors that are interconnected and highly distributed. Neural network processes by accepting inputs, $x(n)$, which are then multiplied by a set of weights, $w(n)$. The neurons then nonlinearly transform the sum of the weighted inputs, by means of a transfer function, $f$, into an output value $a$, as shown in (1). The output of a neuron, thus, depends on the neuron's input and on its transfer function. Sometimes a bias, $b$, is also added to the network. The bias is then regarded as a weight with a constant input of 1 [29]

$$
a=f\left(\sum w p\right) \quad \text { or } \quad a=f\left(\sum w p+b\right) .
$$

In general, a neural network model is trained to reach from a particular input to a desired target output until 


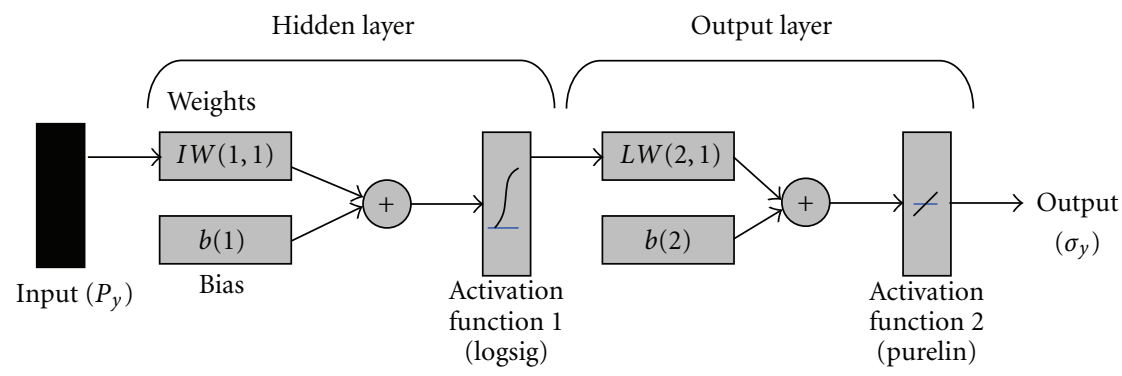

Figure 2: Multilayer network architecture used in the present study.

the network output matches with that of the target. Hence, the neural network can learn all the systems. This system of learning is known as supervised learning. The learning ability of a neural network depends on its architecture and applied algorithmic method during the training. In addition, training procedure can be ceased if the network output reaches close enough to the desired/actual output. Thereafter, the network is ready to produce outputs based on new input parameters that are not used during the learning procedure.

Multilayer feed forward networks are one of the corner stones of research in ANN. In this, the neurons are ordered in layers, with an input set, hidden set and an output set of neurons. The information (data) contained in the input layer is mapped to the output layers through the hidden layers. Each unit can send its output to the units on the higher layer only and receive its input from the lower layer.

The neural network used belongs to the class of multilayer perceptrons or feed-forward neural networks as shown in Figure 2. The two-layered neural network model had "logsig" as activation function in its first layer, that is, hidden layer, and the second layer has "purelin" as its activation function. The number of neurons in the hidden layer varied from 50 to 100 to achieve the optimal network model, whereas the number of neurons in the output layer was 1 . In the present study, training of neural network is carried out using MATLAB version 6.5.

The prediction of yield strength using neural network is made using the load-elongation curve obtained from the miniature test on dumb-bell specimen as shown in Figure 3. In the present investigation, the study has been conducted in various types of steels, that is, chromium steel (H11), low carbon steel (LC) and medium carbon steel (MC), as well as an aluminum alloy (AR66). The compositions of above materials are presented in Table 1. The Al-alloy was in optimum aged condition whereas the various steels were in normalized condition.

\section{Prediction of Yield Strength}

The yield strength prediction scheme used in the present study is shown in Figure 4. The procedure for the evaluation of yield strength using a miniature specimen test along with the neural network model is delineated in a stepwise manner as follows.

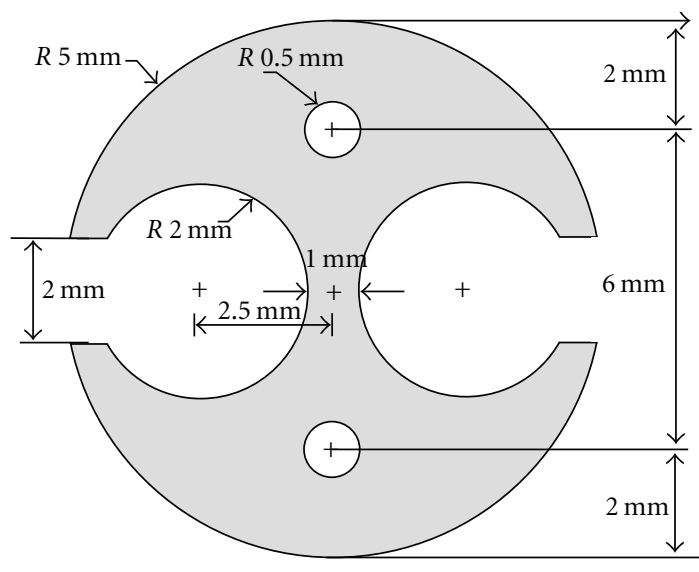

FIGURE 3: Configuration of the miniature dumb-bell specimen.

4.1. Input to the Neural Network. The neural network model for the prediction of yield stress is trained by providing the yield load (breakaway load) $P_{y}$ as the input and yield stress as target output for different materials. The yield load is obtained from the load-elongation curve of miniature test on dumb-bell shaped specimens. A typical load-elongation diagram for the specimen from the Al-alloy (AR66) is shown in Figure 5. Different data points on the loadelongation diagram are identified as $\left(u_{i}, f_{i}\right)$, where $u_{i}$ is the elongation in the specimen due to the corresponding tensile force $f_{i}$ at the $i$ th data point. These data points are obtained directly from the miniature test. Similarly, the load-elongation diagrams were obtained for all other materials considered in the present study. Whenever the distinct yield point is not appearing in the load-elongation curve, yield load is obtained as yield offset corresponding to $0.2 \%$ of the elongation corresponding to the maximum load as shown in Figure 6. The load at breakaway $\left(P_{y}\right)$ obtained from miniature test load-elongation curve for the materials considered in the present study is shown in Table 2.

4.2. Preparation of Database. (a) The database required for the training of ANN was prepared by performing experimental miniature tests on the materials for which the values of yield strength are already known. The experimental load-elongation curves from the miniature test are obtained for all the materials. From the miniature test load-elongation curve, the values of breakaway load are obtained for all the 
TABle 1: The chemical compositions (Wt\%) of steels used in the present study.

\begin{tabular}{lccccccccccc}
\hline Material & $\mathrm{C}$ & $\mathrm{Mn}$ & $\mathrm{Si}$ & $\mathrm{Zn}$ & $\mathrm{Ni}$ & $\mathrm{Cr}$ & $\mathrm{Mo}$ & $\mathrm{Al}$ & $\mathrm{Cu}$ & $\mathrm{Zr}$ & $\mathrm{Fe}$ \\
\hline Aluminum alloy (AR66) & 0.0 & 0.0 & 0.0 & 6.3 & 0.0 & 0.0 & 0.0 & Rest & 1.55 & 0.14 & 0 \\
Chromium steel (H11) & 0.36 & 0.4 & 1.00 & 0.0 & 0.0 & 5.0 & 1.1 & 0.0 & 0.0 & 0.0 & Rest \\
Medium carbon steel (MC) & 0.35 & 0.68 & 0.15 & 0.0 & 0.01 & 0.02 & 0.02 & 0.01 & 0.03 & 0.0 & Rest \\
Low carbon steel (LC) & 0.19 & 0.44 & 0.14 & 0 & 0.15 & 0.1 & 0.04 & 0.02 & 0.13 & 0.01 & Rest \\
\hline
\end{tabular}
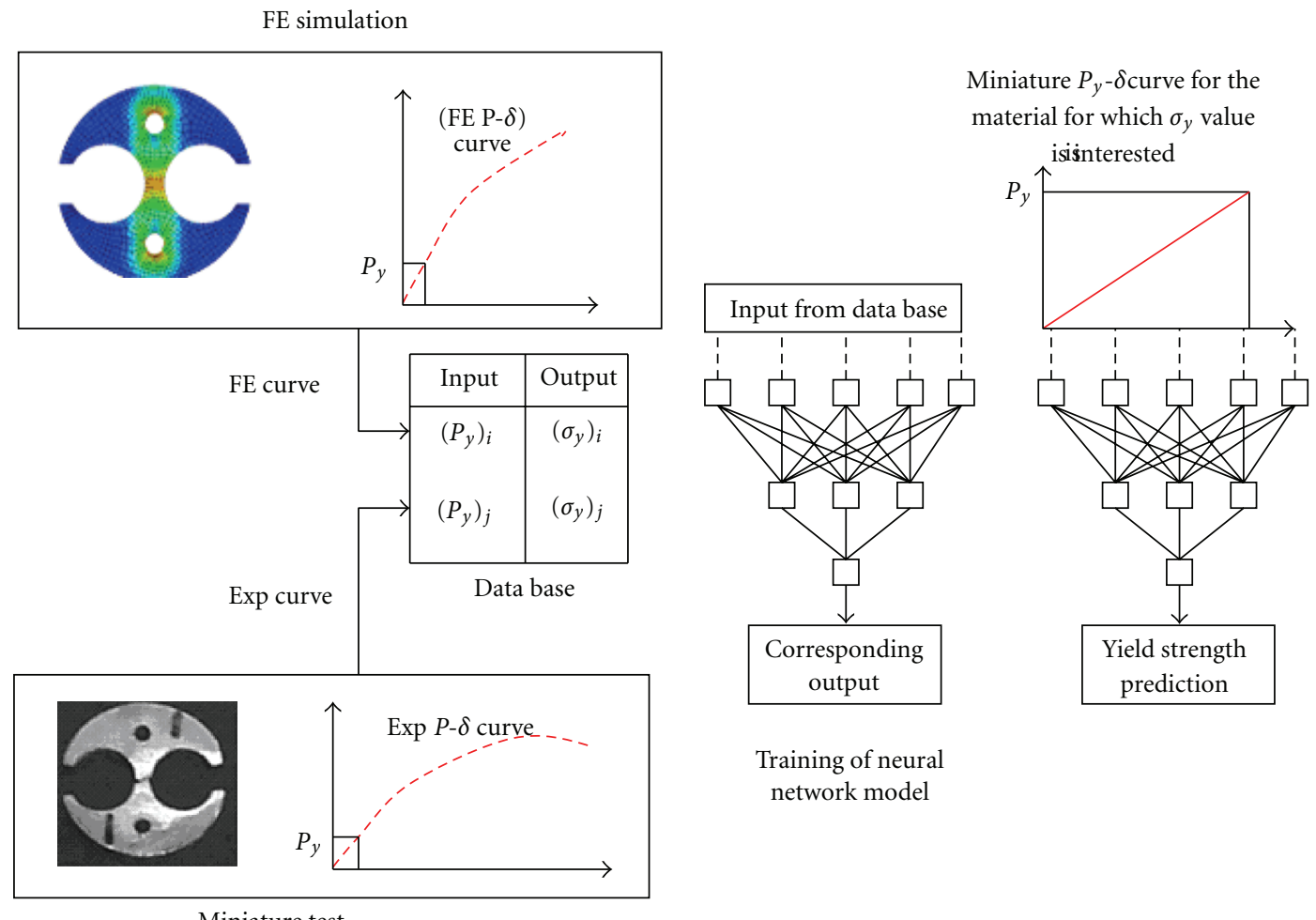

Training of neural network model

FIgURE 4: The scheme of material property prediction.

TABle 2: Load at breakaway point $\left(P_{y}\right)$ for materials in present investigation.

\begin{tabular}{lcc}
\hline Sl. no. & Materials & $\begin{array}{c}\text { Load at breakaway } \\
\left(P_{y}\right) \text { in N }\end{array}$ \\
\hline$(1)$ & AR66 Al-alloy & 129 \\
$(2)$ & H11 steel & 110 \\
$(3)$ & LC steel & 82 \\
$(4)$ & MC steel & 69 \\
\hline
\end{tabular}

materials considered in the study and are denoted as $\left(P_{y}\right)_{i}$ and the corresponding yield strength values of the material are denoted as $\left(\sigma_{y}\right)_{i}$ as shown in Figure 4. They are stored in the database, where the subscript $i$ varies from 1 to $N$ $(N=$ number of different materials on which miniature test is performed).

(b) Apart from the experimental data, also data were collected for various materials from the available literature. The collected data constitutes tensile properties (such as Young's modulus, yield strength, etc.), true tensile stress-true strain diagram of the materials. Finite element simulation was performed using ABAQUS [30] to obtain FE simulated miniature test load-elongation curve for these materials. The description about the finite element simulation of miniature test is given elsewhere [31]. From the FE simulated miniature test load-elongation curves, the values of breakaway load were obtained and are denoted as $\left(P_{y}\right)_{j}$, and the corresponding yield strength values of the material are denoted as $\left(\sigma_{y}\right)_{j}$ (see Figure 4$)$, where $j$ varies from 1 to $M(M=$ number of different materials on which FE simulation of miniature test is performed). Now, the database contains number of load at breakaway and corresponding yield strength values of different materials. Out of the available data $75 \%$ data were used for training the neural network and the remaining were used for testing the network.

4.3. Training of Neural Network. Neural network was trained by using the data stored in the database as described above. The inputs to the neural network are the breakaway load point $\left(P_{y}\right)$ from the database and the corresponding yield strength values are the target output to the neural network. 


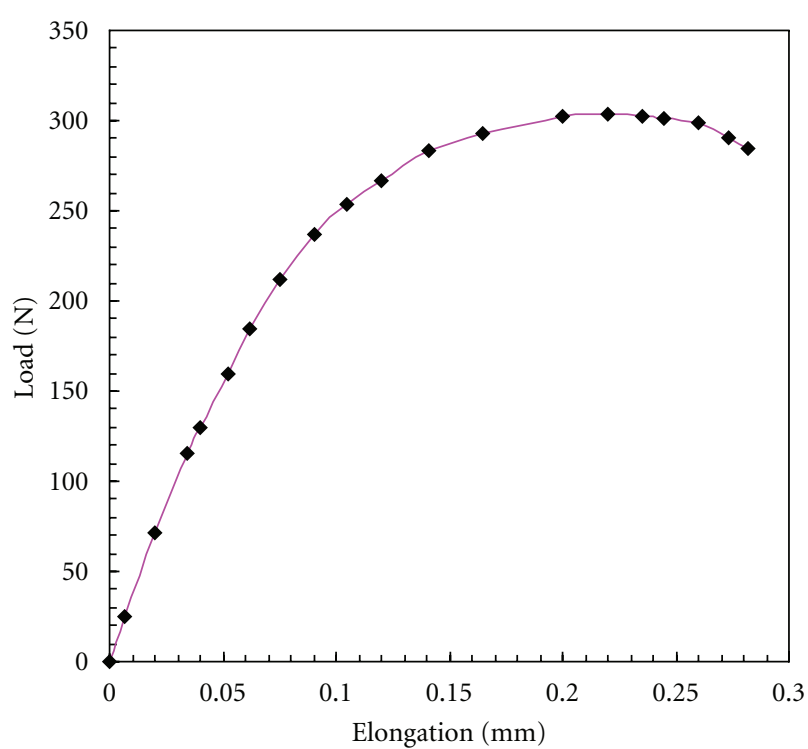

FIGURE 5: Load-elongation diagram for the miniature test on specimen from AR66 Al-alloy.

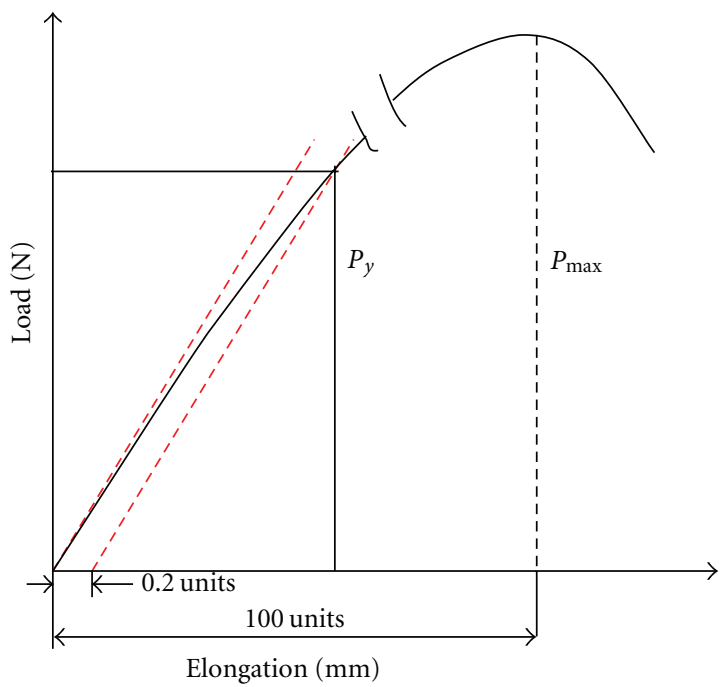

Figure 6: Procedure to find yield load from load-elongation curve from the miniature test.

The network is said to be trained when the output from the ANN matches closely with the target output within the tolerance. The training includes selection of suitable training algorithm for a particular problem. This is a very crucial step. It depends on the complexity of the problem, the number of data points in the training set, the number of weights and biases in the network, and the performance goal as well. The training is done by changing the weights between the layers with an appropriate learning function. In order to find the best suited training algorithm, a series of training algorithms namely Traingdx, Traingda, Trainoss, Trainscg, Traincgp, Traincgb, Traincgf, and Trains based on various transfer functions have been tested for achieving the best

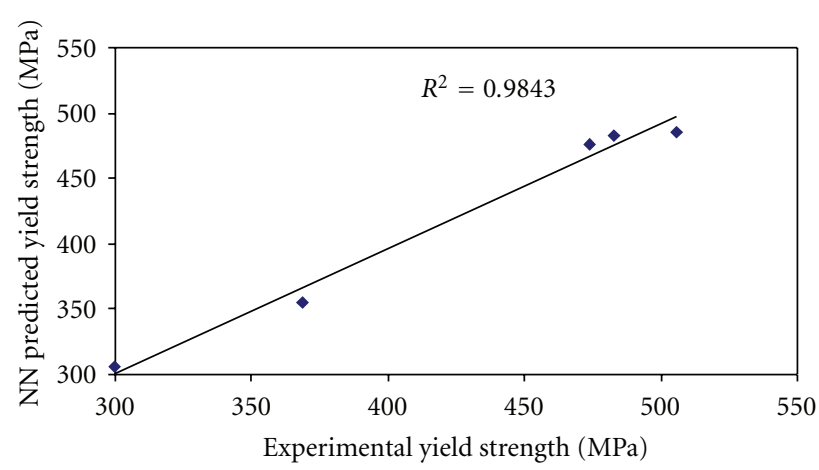

FIGURE 7: The performance of neural network in prediction of yield strength for whole set.

performance goal, that is, mean square error should be minimum and also the computational time should be minimum. The following parameters were adjusted: transfer function, optimization technique, and the number of neurons in the hidden layer of the network. Efficiency was improved by changing the number of hidden layers and the corresponding transfer function based on the training algorithm. It is noted from [25] that Trainscg algorithm gave best result with lowest mean square error in minimum computational time. The trained network model is now ready for predicting the yield strength value with the use of miniature test load-elongation curve of the desired material.

4.4. Yield Strength Prediction Scheme. With the trained neural network model available, the value of yield strength can be obtained for any material by obtaining miniature test load-elongation curve either through experiment or by FE simulation if the tensile properties such as Young's modulus, yield strength, and true stress-true strain diagram of the material are known. The complete procedure is pictorially presented in Figure 4.

4.5. Performance of the Neural Network. The performance of the neural network is checked for the prediction of yield strength over the whole data set and is shown in Figure 7. The analysis of the network response was plotted in the form of linear regression analysis between the network output (predictions) and the corresponding targets (experimental value) for the whole dataset. The neural network predicted the values to an appreciable degree of accuracy. The correlation coefficient $\left(R^{2}\right)$ value is also shown in Figure 7.

In order to test the effectiveness of the developed neural network model, miniature tests were performed on different alloys chosen for the present study whose chemical composition is given in Table 1. In order to compare the result obtained from neural network, standard uniaxial tensile test also performed on these materials according to the standards ASTM E8-03 [32]. The standard uniaxial tensile test specimens were prepared according to ASTM E8 and tests were carried out using Zwick/250 universal testing machine. A calibrated extensometer was used to measure the elastic and plastic strains. The least count 
TABLE 3: Uniaxial tensile properties of different materials.

\begin{tabular}{|c|c|c|c|c|}
\hline \multirow{2}{*}{ Sl. no } & \multirow{2}{*}{ Materials } & \multicolumn{3}{|c|}{ Different mechanical properties } \\
\hline & & $\begin{array}{l}\text { Young's } \\
\text { modulus } \\
(\mathrm{GPa})\end{array}$ & $\begin{array}{c}\text { Yield } \\
\text { strength } \\
(\mathrm{MPa})\end{array}$ & $\begin{array}{r}\text { Tensile } \\
\text { strength } \\
(\mathrm{MPa})\end{array}$ \\
\hline (1) & AR66 alloy & 70 & 507 & 568 \\
\hline (2) & H11 steel & 195 & 474 & 693 \\
\hline (3) & LC steel & 194 & 359 & 511 \\
\hline (4) & MC steel & 200 & 300 & 569 \\
\hline
\end{tabular}

of the extensometer used was $1 \mu \mathrm{m}$. Material properties such as Young's modulus, yield strength, tensile strength, true stress, and true strain diagrams were obtained for all the materials from the uniaxial tensile test. The values of different parameters obtained from the uniaxial tensile test are shown in Table 3.

4.6. Miniature Test Load-Elongation Curve. With the trained neural network model now available, yield strength predictions may be made quickly for new sets of materials without resorting to detailed finite element simulations or the standard testing methods. Such techniques require a lot of time and large volume of the materials for preparing the standard test specimen, which is impractical in inservice structures. In the present case, the miniature test load-elongation diagrams are obtained for the each material by performing miniature tensile test on the specimens prepared from respective materials. From the resulting loadelongation diagrams, the load at breakaway $\left(P_{y}\right)$ is obtained for each material. This load vector is given as input to the neural network. The yield strength obtained for the materials used in the present investigation using the trained neural network are shown in Figure 8. The figure also shows the experimental yield strength from standard tensile test for the respective materials.

Further analysis of the performance accuracy was carried out using statistical analysis of the error of neural network predictions. Neural network predictions are compared to the corresponding experimental values. The relative errors are calculated using the relationship

$$
\text { Error in } \%=\frac{100 *(T-A)}{T} \text {, }
$$

where $T$ is the experimental (measured) output value and $A$ is the predicted value by the neural network. Table 4 shows the error in neural network predicted yield strength value.

It is observed from Table 4 that the ANN predicted yield strength values are in good agreement with those of the experimental values. For AR66 Al-alloy material, the yield strength value predicted by ANN is $484.7 \mathrm{MPa}$, whereas the experimental value is $506 \mathrm{MPa}$. The percentage error in this case is maximum, that is, $4.20 \%$ when compared to other materials. For all other materials, the percentage error is within $4 \%$. So, it can be used to predict the yield strength value of any unknown material by using the output of miniature test on dumb-bell-shaped specimen.

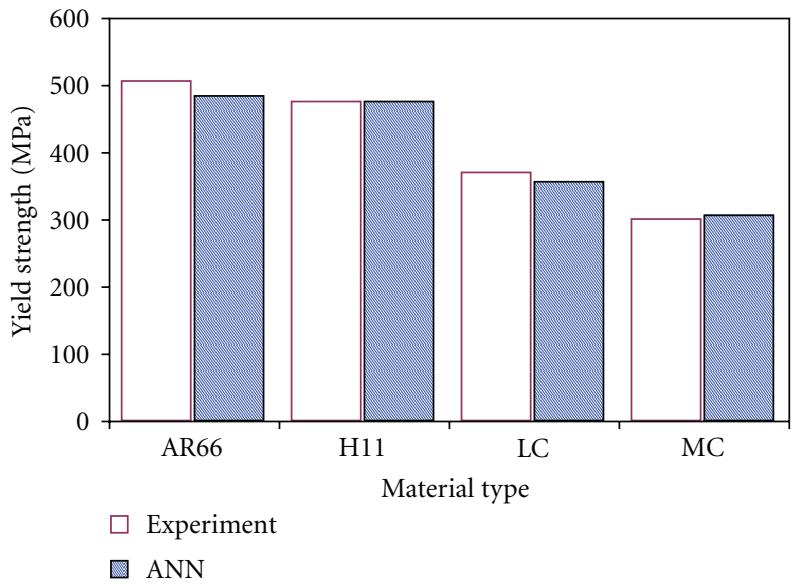

FIGURE 8: Comparison of ANN predicted yield strength with experimental result.

TABLE 4: Error in neural network predicted yield strength.

\begin{tabular}{lc}
\hline Materials & Error in \% \\
\hline AR66 Al-alloy & 4.20 \\
Chromium steel (H11) & 0.40 \\
Low carbon steel (LC) & 3.90 \\
Medium carbon steel (MC) & 1.70 \\
\hline
\end{tabular}

\section{Validation of Neural Network Model}

After testing the proposed neural network model with the data obtained from experimental miniature test of different materials, the trained network is also validated with the data obtained from literatures $[9,33]$. The required neural network input (i.e., the load at breakaway point $P_{y}$ ) for the material were obtained by performing the $2 \mathrm{D}$ finite element simulation of dumb-bell-shaped miniature specimen using the tensile properties as given in the literature. The simulations were performed using ABAQUS.

With the trained neural network model readily available, $P_{y}$ was fed as input to the network and the neural network predicted the yield strength value of the material. The results are presented in Table 5. It is observed that the yield strength predicted by ANN for the die steel is $482.4 \mathrm{MPa}$ in comparison with the experimental evaluated yield strength value of $483 \mathrm{MPa}$, that is, with the error of $0.12 \%$. Similarly, the ANN predicted the yield strength of medium carbon steel as $317.2 \mathrm{MPa}$ with the error of $1.49 \%$ when compared to its experimental value of $322 \mathrm{MPa}$. Thus, the developed neural network model along with miniature test result helped in achieving yield strength value of the materials in efficient way.

\section{Conclusions}

The yield strength value predicted by neural network model with Trainscg algorithm is found to be corroborating well with the standard test results as shown in Figure 8 and Table 5. The yield strength value of AR66 Al-alloy predicted 
TABLE 5: Validation of neural network model in yield strength prediction.

\begin{tabular}{lccc}
\hline Materials & $\begin{array}{c}\text { Exp. yield } \\
\text { strength (MPa) }\end{array}$ & $\begin{array}{c}\text { ANN predicted } \\
\text { yield strength } \\
(\mathrm{MPa})\end{array}$ & Error in \% \\
\hline $\begin{array}{l}\text { Die steel [33] } \\
\text { Medium carbon }\end{array}$ & 483 & 482.4 & 0.12 \\
steel [9] & 322 & 317.2 & 1.49 \\
\hline
\end{tabular}

by ANN is $484.7 \mathrm{MPa}$, whereas the experimental value is $506 \mathrm{MPa}$, for the H11 steel the neural network predicted the yield strength value as $475.9 \mathrm{MPa}$ with an error of $0.40 \%$ as compared to its experimental value that is, $474 \mathrm{MPa}$. Similarly, the yield strength values predicted by the ANN for the low carbon steel and medium carbon steel are 354.6 and $305.1 \mathrm{MPa}$, respectively, with the error of $3.90 \%, 1.70 \%$ and when compared to their respective experimental yield strength values. Also, the present neural network model predicted the yield strength value of the materials taken from the available literature to an appreciable degree with error in their prediction varied from $0.12 \%$ to $1.50 \%$ for various materials. The approach seems to have potential to predict the other mechanical properties of the material, which could be used in remaining life estimation of the costly energy producing plants and other structures.

In addition, the proposed novel dumb-bell-shaped miniature specimen can be prepared from the material taken out noninvasively from the location of interest where adverse conditions are present in the in-service component. The properties of the material at this location of interest can be obtained nondestructively through the approach employed in the present study.

\section{References}

[1] M. E. Haque and K. V. Sudhakar, "ANN back-propagation prediction model for fracture toughness in microalloy steel," International Journal of Fatigue, vol. 24, no. 9, pp. 1003-1010, 2002.

[2] J. R. Foulds and R. Viswanathan, "Determination of the toughness of in-service steam turbine disks using small punch testing," Journal of Materials Engineering and Performance, vol. 10, no. 5, pp. 614-619, 2001.

[3] G. E. Lucas, "Review of small specimen test techniques for irradiation testing," Metallurgical and Materials Transactions A, vol. 21, pp. 1105-1119, 1990.

[4] F. M. Huang, M. L. Hamilton, and G. L. Wire, "Bend testing for miniature disks," Nuclear Technology, vol. 57, pp. 234-242, 1982.

[5] X. Mao and H. Takahashi, "Development of a furtherminiaturized specimen of $3 \mathrm{~mm}$ diameter for Tem disk small punch tests," Journal of Nuclear Materials, vol. 150, no. 1, pp. 42-52, 1987.

[6] X. Mao, M. Saito, and H. Takahashi, "Small punch test to predict ductile fracture toughness JIC $_{\text {IC }}$ and brittle fracture toughness $\mathrm{K}_{\mathrm{IC}}$," Scripta Metallurgica et Materiala, vol. 25, no. 11, pp. 2481-2485, 1991.
[7] P. S. Kullen, H. H. Smith, and D. J. Michel, "The shear punch measurement of the mechanical properties of selected unirradiated and irradiated alloys," Journal of Nuclear Materials, vol. 158, pp. 57-63, 1988.

[8] R. K. Pandey and S. Bhowmick, "Studies on miniature test techniques for the assessment of residual life," in Proceedings of Trends in Mechanical Engineering Education and Research, pp. 542-547, New Delhi, India, 1999.

[9] A. Husain, Determination of mechanical behaviour of materials using miniature specimen test technique and finite element method, Ph.D. thesis, Department of Applied Mechanics, Indian Institute of Technology Delhi, New Delhi, India, 2003.

[10] C. C. Tsao, "Prediction of flank wear of different coated drills for JIS SUS 304 stainless steel using neural network," Journal of Materials Processing Technology, vol. 123, no. 3, pp. 354-360, 2002.

[11] M. S. Ozerdem and S. Kolukisa, "Artificial neural network approach to predict the mechanical properties of $\mathrm{Cu}-\mathrm{Sn}-$ $\mathrm{Pb}-\mathrm{Zn}$-Ni cast alloys," Materials and Design, vol. 30, no. 3, pp. 764-769, 2009.

[12] M. S. Ozerdem and S. Kolukisa, "Artificial Neural Network approach to predict mechanical properties of hot rolled, nonresulfurized, AISI 10xx series carbon steel bars," Journal of Materials Processing Technology, vol. 199, no. 1-3, pp. 437-439, 2008.

[13] S. Forouzan and A. Akbarzadeh, "Prediction of effect of thermo-mechanical parameters on mechanical properties and anisotropy of aluminum alloy AA3004 using artificial neural network," Materials and Design, vol. 28, no. 5, pp. 1678-1684, 2007.

[14] M. Li, X. Liu, and A. Xiong, "Prediction of the mechanical properties of forged TC11 titanium alloy by ANN," Journal of Materials Processing Technology, vol. 121, no. 1, pp. 1-4, 2002.

[15] Z. Guo and W. Sha, "Modelling the correlation between processing parameters and properties of maraging steels using artificial neural network," Computational Materials Science, vol. 29, no. 1, pp. 12-28, 2004.

[16] S. Malinov, W. Sha, and J. J. McKeown, "Modelling the correlation between processing parameters and properties in titanium alloys using artificial neural network," Computational Materials Science, vol. 21, no. 3, pp. 375-394, 2001.

[17] J. McBride, S. Malinov, and W. Sha, "Modelling tensile properties of gamma-based titanium aluminides using artificial neural network," Materials Science and Engineering A, vol. 384, no. 1-2, pp. 129-137, 2004.

[18] C. Z. Huang, L. Zhang, L. He et al., "A study on the prediction of the mechanical properties of a ceramic tool based on an artificial neural network," Journal of Materials Processing Technology, vol. 129, no. 1-3, pp. 399-402, 2002.

[19] Z. Zhang, P. Klein, and K. Friedrich, "Dynamic mechanical properties of PTFE based short carbon fibre reinforced composites: experiment and artificial neural network prediction," Composites Science and Technology, vol. 62, no. 7-8, pp. 10011009, 2002.

[20] Q. Zeng, J. Zu, L. Zhang, and G. Dai, "Designing expert system with artificial neural networks for in situ toughened $\mathrm{Si}_{3} \mathrm{~N}_{4}$," Materials and Design, vol. 23, no. 3, pp. 287-290, 2002.

[21] M. Perzyk and A. W. Kochański, "Prediction of ductile cast iron quality by artificial neural networks," Journal of Materials Processing Technology, vol. 109, no. 3, pp. 305-307, 2001.

[22] P. Myllykoski, J. Larkiola, and J. Nylander, "Development of prediction model for mechanical properties of batch annealed thin steel strip by using artificial neural network modeling," 
Journal of Materials Processing Technology, vol. 60, no. 1-4, pp. 399-404, 1996.

[23] N. Huber and C. Tsakmakis, "Determination of constitutive properties from spherical indentation data using neural networks. Part II: the case of pure kinematic hardening in plasticity laws," Journal of the Mechanics and Physics of Solids, vol. 47, no. 7, pp. 1589-1607, 1999.

[24] M. Abendroth and M. Kuna, "Determination of deformation and failure properties of ductile materials by means of the small punch test and neural networks," Computational Materials Science, vol. 28, no. 3-4, pp. 633-644, 2003.

[25] G. Partheepan, Determination of mechanical properties of materials using newly developed dumb-bell shaped miniature specimen and numerical techniques, Ph.D. thesis, Department of Applied Mechanics, Indian Institute of Technology Delhi, New Delhi, India, 2006.

[26] G. Partheepan, D. K. Sehgal, and R. K. Pandey, "Design and usage of a simple miniature specimen test setup for the evaluation of mechanical properties," International Journal of Microstructure and Materials Properties, vol. 1, no. 1, pp. 3850, 2005.

[27] K. Chakraborty, K. Mehrotra, C. K. Mohan, and S. Ranka, "Forecasting the behavior of multivariate time series using neural networks," Neural Networks, vol. 5, no. 6, pp. 961-970, 1992.

[28] M. Çöl, H. M. Ertunç, and M. Yilmaz, "An artificial neural network model for toughness properties in microalloyed steel in consideration of industrial production conditions," Materials and Design, vol. 28, no. 2, pp. 488-495, 2007.

[29] L. Fausett, Fundamentals of Neural Networks: Architectures, Algorithms, and Applications, Prentice-Hall, New Jersey, NJ, USA, 1994.

[30] K. Hibbit and Sorensen Inc., ABAQUS Users Manual I-II version 6.3, USA, 2002.

[31] G. Partheepan, D. K. Sehgal, and R. K. Pandey, "Finite element application to estimate in-service material properties using dumb-bell miniature specimen," in Proceedings of the First International Conference on Advances and Trends of Engineering Materials and Their Applications (AES-ATEMA '07), pp. 369377, Montreal, Canada, August 2007.

[32] ASTM Standard Test Method for Tension Testing of Metallic Materials, E8-03, Annual book of ASTM standard, 2003.

[33] A. Husain, D. K. Sehgal, and R. K. Pandey, "An inverse finite element procedure for the determination of constitutive tensile behavior of materials using miniature specimen," Computational Materials Science, vol. 31, no. 1-2, pp. 84-92, 2004. 

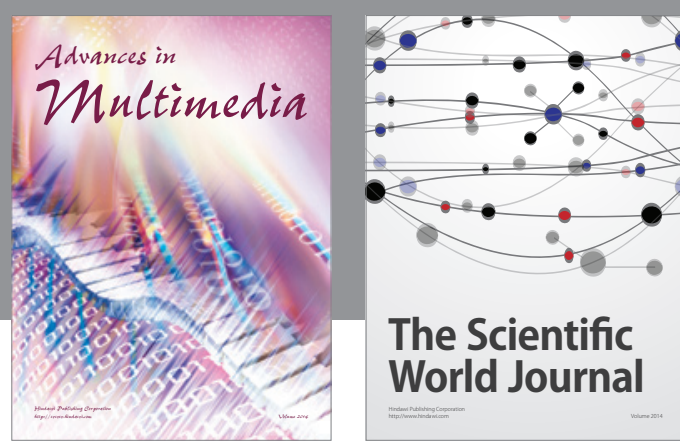

The Scientific World Journal
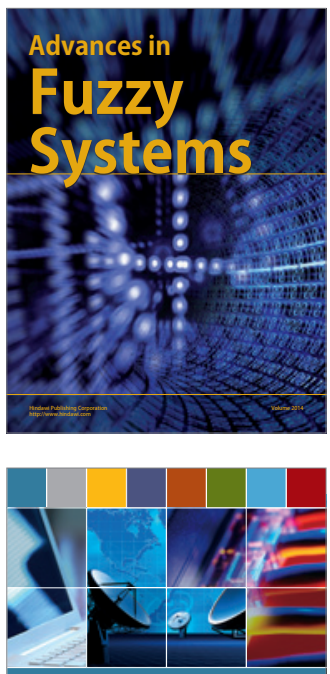

Computer Networks and Communications
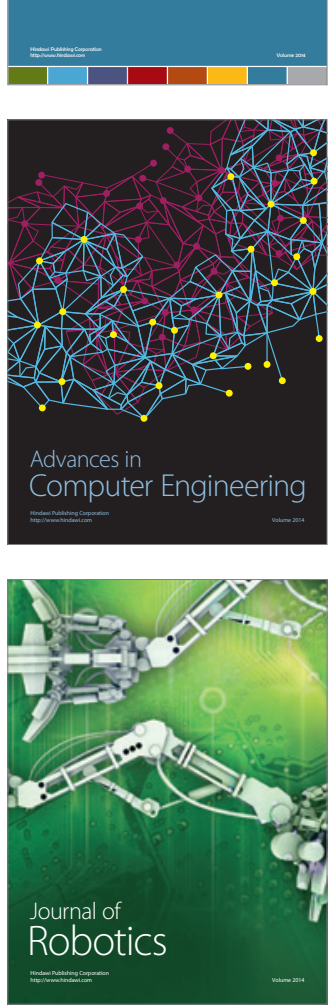
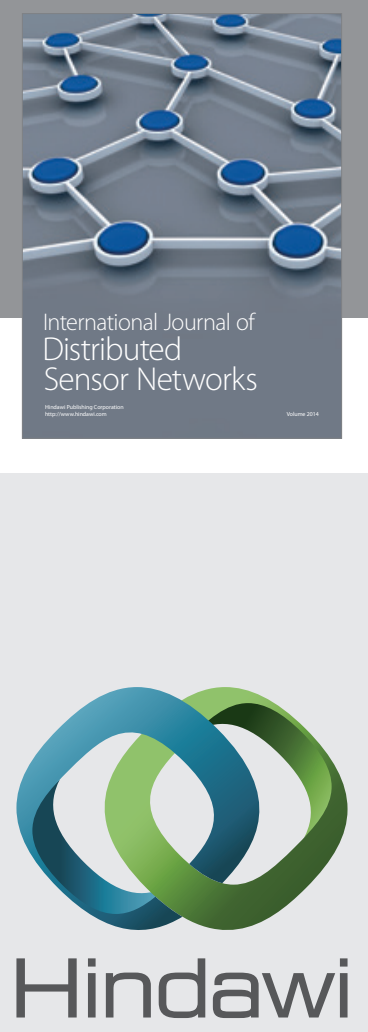

Submit your manuscripts at

http://www.hindawi.com
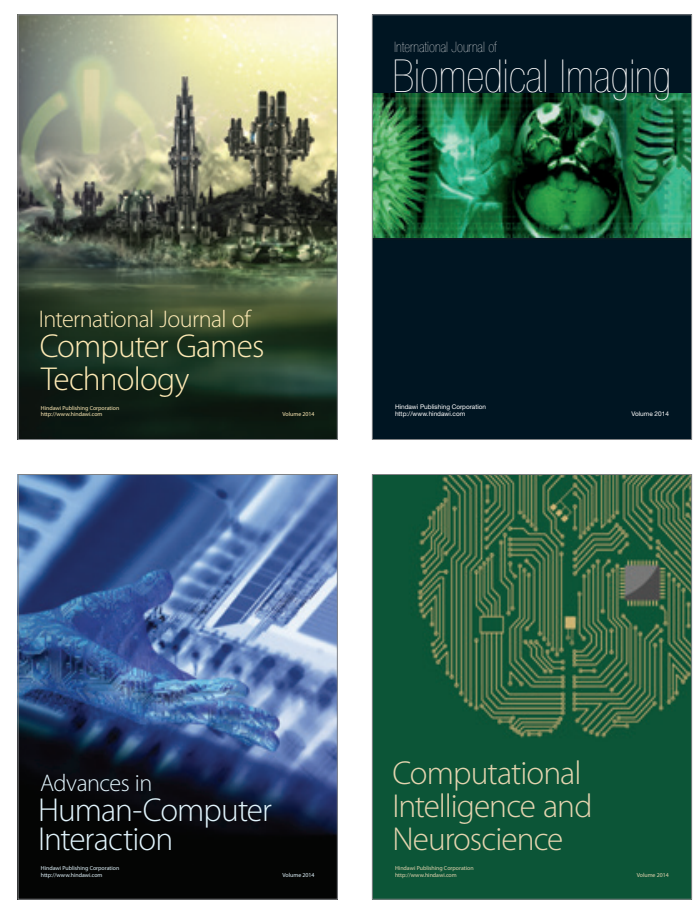
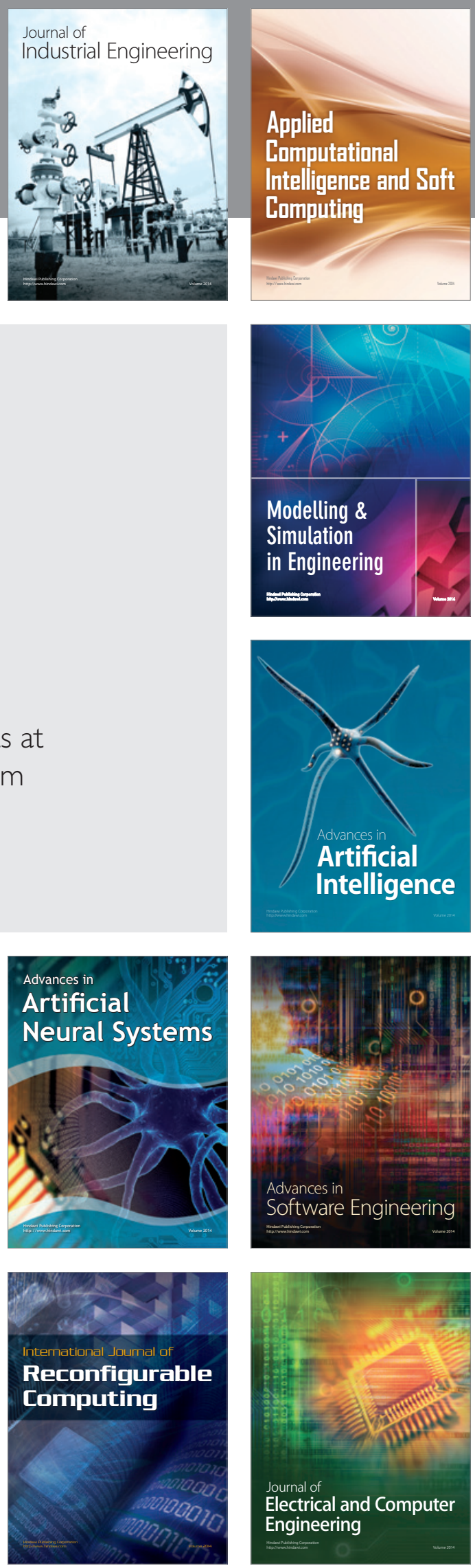